\title{
Quantum Mechanical Hysteresis and the Electron Transfer Problem
}

\author{
P. G. Etchegoin \\ The McDiarmid Institute for Advanced Materials and Nanotechnology \\ School of Chemical and Physical Sciences \\ Victoria University of Wellington \\ PO Box 600 Wellington, New Zealand
}

(Dated: November 5, 2018)

\begin{abstract}
We study a simple quantum mechanical symmetric donor-acceptor model for electron transfer (ET) with coupling to internal deformations. The model contains several basic properties found in biological ET in enzymes and photosynthetic centers; it produces tunnelling with hysteresis thus providing a simple explanation for the slowness of the reversed rate and the near $100 \%$ efficiency of ET in many biological systems. The model also provides a conceptual framework for the development of molecular electronics memory elements based on electrostatic architectures.
\end{abstract}

PACS numbers: 82.30.Fi; 82.39.Jn; 05.45.-a; Keywords: Tunnelling, Monte Carlo, Electron transfer, Biological Processes

The physics of electron transfer (ET) is of paramount importance in many fundamental aspects of molecular biology, from photosynthesis [1], to mitochondrial ET, to many control reactions and signalling through the cell membrane 2, 3, 4]. Being a sophisticated quantum mechanical tunnelling process in itself, the ET problem includes many aspects of coupling to vibrational degrees of freedom, tunnelling control through external variables, and non-reversibility aspects [2]. After the pioneering work of Marcus [5] in the electrochemical aspects of ET for redox reactions in solution, the problem has seen several times renewed interest in both semiclassical and quantum mechanical (QM) aspects. This is an area where there is a strong interplay between QM and important biological effects at the molecular level.

In addition, there is considerable evidence now on the importance of nonlinear contributions to many important dynamical aspects of molecular biology. DNA dynamics and denaturation [ 6 , 7] and signal transduction and coordination of events during the DNA readout by proteins $[8,9]$ have both been ascribed to nonlinearities. The nonlinear character of the hydrogen bonding potential among DNA bases has been known for a very long time 4, 10, 11]. But nonlinearities in biology are believed to play also a substantial role in ET and energy transfer. Resonant dipole-dipole interactions of the amide I band combined with structural deformations in alpha-helical proteins have been proposed for a very long time as a possible explanation for energy transport from the hydrolysis of ATP 12]. There is experimental evidence that amide I vibrations are coupled nonlinearly; an example being their unusually long life in simple proteins [13]. The coupling of carriers to conformational degrees of freedom has been suggested also to exist in connection with ET from the active prosthetic groups inside cytochromes [4]. There

*Electronic address: Pablo.Etchegoin@vuw.ac.nz seems to be, in addition, substantial evidence that a nonlinear coupling is required to explain the so-called gated ET reactions between quinones in the photosynthetic reaction centers [14]. Knapp and coworkers [15] were among the first to realize the importance of both nonlinearities and Davidov-like solitons [4] in the ET problem. They argued that the construction of the initial and final wavefunctions in terms of solitary states leads automatically to localization for a symmetric donor-acceptor system, a very desirable model property in many cases, as we shall explain later.

The brief outline given above highlights the fact that there is a case in many real biological systems for the study of QM tunnelling properties in the presence of intrinsic nonlinearities. To this end, there are two possible options, either we study the specific non-linear coupling of a given case with all its peculiarities and details, as done in Ref. 14 for ET between quinones in the photosynthetic reaction center, or we study a skeletal model which contains the essential elements of the problem and try to draw general qualitative conclusions from its physical behavior. We shall adopt the latter view here to highlight a specific property of the tunnelling process in the presence of nonlinearities; i.e. the presence of hysteresis in a symmetric donor-acceptor ET system.

Davydov's approach to electron-lattice deformation coupling in biological systems [4] and its variants [15] lead always to an effective Hamiltonian of the non-linear Schrödinger equation (NLS) type. The nonlinearity in the equation comes from a hidden adiabatic coupling to an external interaction (the deformation) which is factored out from the dynamics in the form of an effective self-consistent potential. The Hamiltonian is always of the self-localizing type, i.e. with a negative nonlinear potential. We have study very recently two models with this property in the framework of the ET problem 16]. The simplest possible discrete symmetric donor-acceptor ET system, including a nonlinear coupling of the Davydov type, is the two-sites nonlinear Hubbard (NLH) model, 
given in second quantization by the Hamiltonian:

$$
\begin{aligned}
\hat{H}= & \epsilon_{1} c_{1}^{\dagger} c_{1}+\epsilon_{2} c_{2}^{\dagger} c_{2}+t\left(c_{2}^{\dagger} c_{1}+c_{1}^{\dagger} c_{2}\right)- \\
& {\left[U\left\langle n_{1}\right\rangle c_{1}^{\dagger} c_{1}+U\left\langle n_{2}\right\rangle c_{2}^{\dagger} c_{2}\right], }
\end{aligned}
$$

where $\left\langle n_{i}\right\rangle=\left\langle c_{i}^{\dagger} c_{i}\right\rangle$, and 1 (2) refer to the donor (acceptor). The Hubbard-like terms $\propto U>0$ give the necessary (non-linear) self-trapping potential and $\epsilon_{i}$ and $t$ are the site energies and overlap (hoping) integral between the two sites, respectively. This would be the case of tunnelling between two equivalent sites with strong polaronic coupling. This model, and variants of it including dissipation, has been extensively studied for many years by Kenkre and coworkers 17, 18, 19, 20] who coined the term quantum nonlinear dimer. The objective of this Letter is to focus on a specific property of the tunnelling between the two sites in this model, namely: the presence of hysteresis. We shall then speculate on situations where this property may be playing a role in ET with nonlinear couplings. We review very briefly a few of its properties discussed in Ref. 16. The ground state can be sought by a Monte Carlo (MC) simulated annealing in $\left[z_{1}-z_{2}\right]$ space, were $z_{1}$ and $z_{2}$ are the complex coefficients of the ground state wavefunction for the amplitude of the electron at the donor and acceptor sites, respectively, i.e.

$$
\Psi_{G}=\left(\begin{array}{c}
z_{1} \\
z_{2}
\end{array}\right)
$$

with $\left|z_{1}\right|^{2}+\left|z_{2}\right|^{2}=1$. A good measure of the asymmetry in the electronic density of the two sites is the magnitude

$$
\left.S \equiv|| z_{1}\right|^{2}-\left|z_{2}\right|^{2} \mid,
$$

which gives the absolute value of the difference between the probability densities for donor and acceptor. Figure 11 is the calculated $S$ as a function of $t / U$ in the ground state of a symmetric donor-acceptor system with $\epsilon_{1}=$ $\epsilon_{2}=0$. Alternatively, the minimization of (11) subject to the normalization condition renders $S=\sqrt{1-(t / U)^{2}}$ for $t / U<1$ and $S=0$ for $t / U>1$, as seen in Fig. [1 If $t / U$ is large (small $U$ ) the ground state is symmetric, i.e. $S=0$, and the electron is equally shared between both sites. Below a certain value of $t / U$, the system displays a spontaneous symmetry breaking in the ground state, as discussed in Ref. 16. From there on the system profits from accumulating the wavefunction in one of the sites and $S \rightarrow 1$ as $t / U \rightarrow 0$. This is the localization in the symmetric donor-acceptor system mentioned by Knapp et. al. 15].

The question we raise now is how the electron behaves when we start varying the energy $\epsilon_{i}$ of the levels externally. We shall show that the intrinsic nonlinearity of
Davydov-type models for ET introduces hysteresis in the quantum mechanical tunnelling.

The qualitative reason is relatively easy to understand and is shown in Fig. 22 Let us assume that $U$ is big $(t / U$ small) and the electron is mainly trapped on the left. We now vary the potential of $\epsilon_{2}$ making it lower than $\epsilon_{1}$. Under normal circumstances, the electron would tunnel immediately to the right. However this might not be the case in the presence of a nonlinearity, because the energy of the electron localized on the left is of the order of $\sim$ $\epsilon_{1}-U$, and this could be less than $\epsilon_{2}$, i.e. the nonlinearity is holding the electron in its original place. There is a memory effect, accordingly, which cannot exist in any linear, adiabatic, quantum mechanical system. The same holds for the reverse situation if we raise $\epsilon_{2}$ above $\epsilon_{1}$ once the electron is localized on the right. By fixing $\epsilon_{1}$, the tunnelling events left $\rightarrow$ right or right $\rightarrow$ left occur at two different values of $\epsilon_{2}$, producing an intrinsic hysteresis in a very simple QM system.

Mathematically, however, the property of hysteresis with a nonlinear Hamiltonian is in general difficult to prove. One could argue that in Fig. 2] a gradual spread of the wavefunction from left to right is produced until the electron is fully localized on the right. And the opposite might hold on the reversed bias situation, thus producing a crossover without hysteresis. The way to prove the existence of hysteresis with a sudden transition between the two situations is by properly solving the model. Notwithstanding, a perturbative approach is doomed to fail. The reason is that a localized transition from donor to acceptor is more of a cooperative effect. In what follows we concentrate on the problem of the stability of the wavefunction given an initial (localized) starting condition and a bias between $\epsilon_{1}$ and $\epsilon_{2}$.

It is advantageous to change to a continuous version of the model in Fig. 22 We treat a model with a similar property (spontaneous symmetry breaking) that is related to (1): the nonlinear particle in a box. In Ref. 16 it was shown that a quantum mechanical particle in a box obeying the NLS equation undergoes an spontaneous symmetry breaking in the probability density for a sufficiently large value of the nonlinear potential. The particle is confined either on the left or the right of the box and it is possible to produce tunnelling between the two situations under the presence of an external stochastic potential. The NLS, which is the equivalent to (11) for a continuous system, reads

$$
\gamma \nabla^{2} \psi(\underline{\mathbf{r}})+\beta|\psi(\underline{\mathbf{r}})|^{2} \psi(\underline{\mathbf{r}})=E \psi(\underline{\mathbf{r}}),
$$

where $\psi(\underline{\mathbf{r}})$ is subject to the boundary conditions $\left.\psi(\underline{\mathbf{r}})\right|_{ \pm a / 2}=0$, with $a=$ size of the box. $E$ is the energy and $\gamma<0, \beta<0$ are the kinetic energy and self-trapping potential parameters, respectively. The relevant magnitude is the ratio $\rho=|\gamma / \beta|$, fixing the relative strength of both. For small $\rho$, an spontaneous self-localization of the wavefunction either to the left or the right of the box occurs [16], both sides playing the equivalent roles of the 
donor-acceptor sites in (11). The continuous model (4) allows the inclusion of an electric field competing with the stability of the self-localized ground state. The presence of an external potential, in the one-dimensional problem, is added to (4) through an additional term on the righthand side of the form:

$$
V_{0}\left(1-\frac{2 x}{a}\right) \psi(x)
$$

where $V_{0}$ is a constant, and $\underline{\mathbf{r}} \equiv x$ in this case. This potential produces a linear slope (constant electric field) form one end $(x=-a / 2)$ to the other $(x=+a / 2)$ of the box.

In addition, the stability of the wavefunction can be tested with the aid of the Fourier Monte-Carlo (FMC) method developed in Ref. 16. We review here very briefly the basic concepts involved, while leaving details to the references 16. The wavefunction can always be expressed by a Fourier series of arbitrary length of the form

$$
\psi(\underline{\mathbf{r}})=\sqrt{\frac{(2 / a)}{\sum_{n}\left(a_{n}^{2}+b_{n}^{2}\right)}} \sum_{n}\left[a_{n} \cos \left(\underline{\mathbf{k}}_{n}^{c} \cdot \underline{\mathbf{r}}\right)+b_{n} \sin \left(\underline{\mathbf{k}}_{n}^{s} \cdot \underline{\mathbf{r}}\right)\right],
$$

with $\underline{\mathbf{k}}_{n}^{s}=2 \pi / a(n+1)$ and $\underline{\mathbf{k}}_{n}^{c}=2 \pi / a(n+1 / 2)$. $a_{n}-b_{n}$ are Fourier coefficients and $\psi(\underline{\mathbf{r}})$ satisfies automatically both the boundary and normalization conditions. The basic idea of the FMC method is that the ground state is found through a simulated annealing (as a function of an artificial temperature $T$ ) in Fourier coefficient space $a_{n}-b_{n}$.

Once the ground state is found for $T=0$, the potential can be changed and the evolution of the ground state monitored for small finite temperature $T_{0}$. It is understood that the particle follows adiabatically the changes in potential. The role of $T_{0}$ is that of a stochastic perturbation testing the stability of the ground state; $T_{0}$ has to be smaller than the energy of any metastability of the system in the presence of nonlinearities. The convergence of the method is tested by doubling the number of coefficients and comparing the quantitative results 16.

Figure 3] shows the result for a full cycle of the electric field in both directions. The parameters of the simulation are given in caption. We start with the particle confined on the left (which we arbitrarily call donor) and we follow the evolution of the wavepacket by monitoring $\langle x\rangle / a=$ $(1 / a) \int_{-a / 2}^{a / 2} x \psi^{2}(x) \mathrm{d} x$ for different potentials in the cycle shown in Fig. 3(a). Figure 3(b) shows a result with the hallmark of hysteresis. As before, the reason is the competition between the intrinsic nonlinearity and the external potential, fixing either the mismatch of the levels in Fig. 20 or the energy difference between left and right in Fig. 3 (b). The transition is not gradual (as it would be without nonlinearity) because the electron does not profit from a reduction in energy by transferring part of its wavefunction to the other side, until a very specific condition in terms of the competition with the correlation energy $U$ is achieved.

By having a model that combines basic aspect which are know to exist in real ET systems, we can now go one step backwards and contemplate those situations where this effect might be playing a role. Control of ET is decisive in many biological functions; the canonical example being ET in the mitochondrial chain, which must stop when ADP is all phosphorylated. For more than 30 years it has been suggested that the energy matching between donor and acceptor could provide a mechanism of ET control 21, 22]. In general terms, this ability of control has to be compatible with the slowness of the reverse rate responsible for the near $100 \%$ quantum efficiency observed, for example, in the photosynthetic reaction centers, and with the presence of couplings to the local environments. These requirements are normally met by the so-called energy-gap law which postulates two different activation barriers for the forward and backward ET reactions, in the spirit of Marcus theory [1, 2, 5]. It has been also known for a long time that simplified invitro model systems 23 invariably show similar or larger rates for the backwards recombination. It seems then that vibronic nonlinear couplings to the environment are essential to understand the efficiency of the ET mechanism, and that evolution through natural selection has engineered a favorable combination of nonlinearity and control capabilities.

It is clear, accordingly, that a basic model like the one presented here satisfies several of these conditions simultaneously. The presence of QM-hysteresis is a key issue that has been overlooked in the opinion of the present author, and provides a very fine mechanism of control even in the presence of broadening of the electronic states through vibronic coupling at the donor and acceptor sites. In addition, the back-reaction problem is automatically solved by the same token, without having to resort to ideas which are normally more difficult to justify, like the presence of two radically different matrix elements for the forward and backward reaction, respectively 24]. We believe that a model of tunnelling between donor and acceptor in the spirit of the models proposed by Davydov [4] and Knapp 15, contain the essential qualitative ingredients to explain simultaneously both control and efficiency of real ET system found in biological systems. The main objective of the present paper is then achieved in Fig. [3] i.e. to demonstrate the existence of QM-hysteresis in a very simple model and to highlight its possible relevance in a variety of phenomena associated with ET in biological systems.

The hysteresis cycle in Fig. 3 defines a threshold value for the transition between the two states, in analogy to the coercive field displayed by magnetic systems. In order to show that the hysteresis in the cycle shown in Fig. [3] comes effectively from a competition between the intrinsic nonlinearity of the Hamiltonian and the external

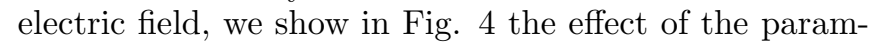


eters of the model on the coercive filed of the transition. Figure 4 shows three examples for a fixed $\gamma$ and three different $\beta$ 's: $-0.1,-0.05$, and 0 . For $\beta=-0.05$ the cycle has narrowed down to a very small region around the origin. The system still profits from accumulating the probability density on the left or the right of the box, but jumps immediately form one situation to the other under the slightest perturbation. The Fourier-MC method has problems to define the exact width of the transition in this case, because the perturbation parameter $T_{0}$ can be comparable to the small barrier separating the two situations. For $\beta=0$, the particle follows smoothly the external electric field. The S-shaped average coordinate of the probability distribution is due now to a competition between the boundary conditions on both sides and the force exerted by the electric field. The wave-packet follows the field linearly at very low fields, but as soon as the displacement from the center is large enough, the boundary conditions on the left or right of the box limit the response to the field; thus achieving saturation in the displacement. These results prove that the nonlinear bistable behavior in Fig. 3 is, effectively, a competition between nonlinearity and external fields in the model.

On more speculative grounds, however, the existence of simple QM-systems with tunnelling hysteresis at the molecular level should also be of interest in other problems. For example, in the field of molecular electronics (ME) 25] where decisive steps have been taken towards the production of logic devices based on the change of electronic properties by single molecules. Charge storage in a self-assembled molecular device has been demonstrated already 26]; the device works by storing a high or low conductivity state achieved by the addition or subtraction of an electron. But the contact problem of organic molecules with metals remains an outstanding problem in $\mathrm{ME}$, and alternatives have been proposed based on the so-called quantum cellular automata (QCA) and electrostatic architectures 25, 27]. The QCA approach has received considerable attention in $\mathrm{ME}$ as an alternative to conventional electronic devices due to its potential in reducing power dissipation by avoiding external currents 25. It is also an interesting conceptual shift from conventional electronic design. After all, the most sophisticated machines known in nature, which are living organism, work mainly not through changes in conductivity and currents but rather through electrostatic and van der Waals interactions.

Even though the technology to achieve ME and QCA devices is only beginning to appear, the results in this paper suggest that organic symmetric donor-acceptor pairs with strong polaronic coupling, and external control by an electric field, are excellent candidates for the ultimate single-molecule dynamic random access memory (RAM) device.

Partial support from EPRSC (UK) at Imperial College London (where part of the work has been done) is gratefully acknowledged.
[1] D. O. Hall and K. Rao, Photosynthesis (Studies in Biology) (Cambridge University Press, Cambridge, 1999).

[2] D. DeVault, Quantum-Mechanical Tunnelling in Biological Systems (Cambridge University Press, Cambridge, 1984), and references therein.

[3] M. V. Volkenstein, Molecular Biophysics (Academic Press, New York, 1977).

[4] A. S. Davydov, Biology and Quantum Mechanics(Pergamon Press, Oxford, 1982).

[5] R. A. Marcus, J. Chem. Phys. 43, 679 (1965), and R. A. Marcus in Light-induced Charge Separation in Biology and Chemistry, edited by H. Gerischer and J. J. Katz (Verlag Chemie, Berlin, 1979), p. 15.

[6] M. Peyrard and A. R. Bishop, Phys. Rev. Lett. 62, 2755 (1989).

[7] Y. Zhang, W. Zheng, J. Liu, and Y. Z. Chen, Phys. Rev. E 56, 7100 (1997).

[8] L. V. Yakushevich, Nonlinear Physics of DNA(Wiley, New York, 1998).

[9] M. Salerno, Phys. Rev. A 44, 5292 (1991).

[10] F. Zhang and M. A. Collins, Phys. Rev. A 52, 4217 (1995).

[11] Y. Z. Chen and E. W. Prohofsky, Phys. Rev. E 49, 3444 (1994).

[12] A. S. Davydov and N. I. Kislukha, Phys. Status Solidi (b) 75, 735 (1976); ibid. 59, 465 (1973).

[13] A. Xie, L. van der Meer, W. IIoff, and R. Austin, Phys. Rev. Lett. 84, 5435 (2000).
[14] B. Rabenstein, G. M. Ullmann, and E. W. Knapp, Biochemistry 39, 10487 (2000).

[15] E. W. Knapp and S. F. Fischer, J. Chem. Phys. 90, 354 (1989).

[16] P. Etchegoin, Chem. Phys. 297, 245 (2004).

[17] G. P. Tsironis and V. M. Kenkre, Phys. Lett. A 127, 209 (1988).

[18] V. M. Kenkre and D. K. Campbell, Phys. Rev. B 34, 4959 (1986).

[19] M. Kus and V. M. Kenkre, Physica D 79, 409 (1994).

[20] V. M. Kenkre, M. F. Jorgensen, and P. L. Christiansen, Physica D 90, 280 (1996).

[21] D. DeVault, J. H. Parkes, and B. Chance, Nature 215, 642 (1967).

[22] L. A. Blumenfeld and D. S. Chernavskii, J. Theor. Bio. 39, 1 (1973).

[23] R. E. Blankenship and W. W. Parson, in Photosynthesis in Relation to Model Systems, edited by J. Barber (Elsevier/North Holland, Amsterdam, 1979), p. 71.

[24] J. Jortner, J. Am. Chem. Soc. 102, 6676 (1980).

[25] J. M. Tour, Molecular Electronics (World Scientific, Singapore, 2003).

[26] J. Chen, W. Wang, M. A. Reed, A. M. Rawlett, D. W. Price, and J. M. Tour, Appl. Phys. Lett. 77, 1224 (2000).

[27] G. L. Snider, A. O. Orlov, I. Amlani, X. Zuo, G. H. Bernstein, C. S. Lent, J. L. Merz, and W. Porod, J. Appl. Phys. 85, 4283 (1999). 


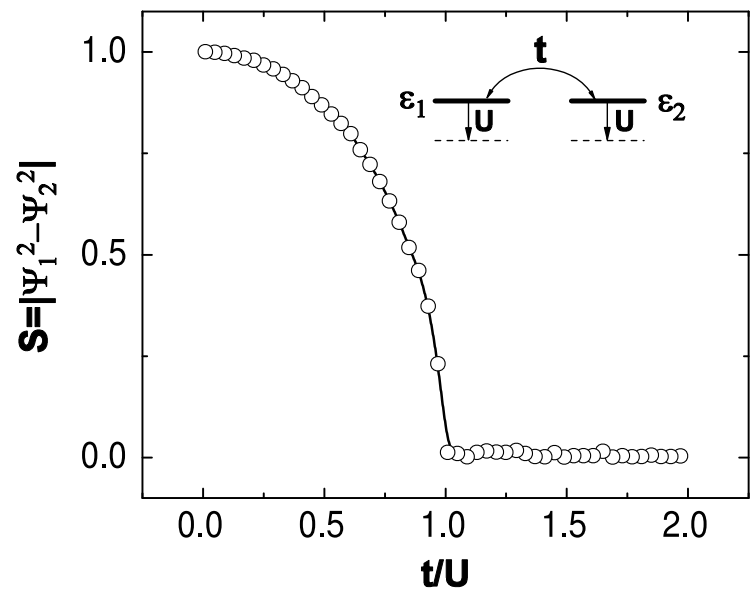

FIG. 1: Asymmetry parameter $S$ (Eqn. 3) for the ground state as a function of $t / U$ for the two-site NLH model (Eqn. 11. A schematic view of the model and its parameters is given in the inset. The calculation is for a symmetric donoracceptor pair with $\epsilon_{1}=\epsilon_{2}=0$. Below $t / U=1$ the system profits by accumulating the electron on one of the sites, thus creating an asymmetric self-localized ground state. 


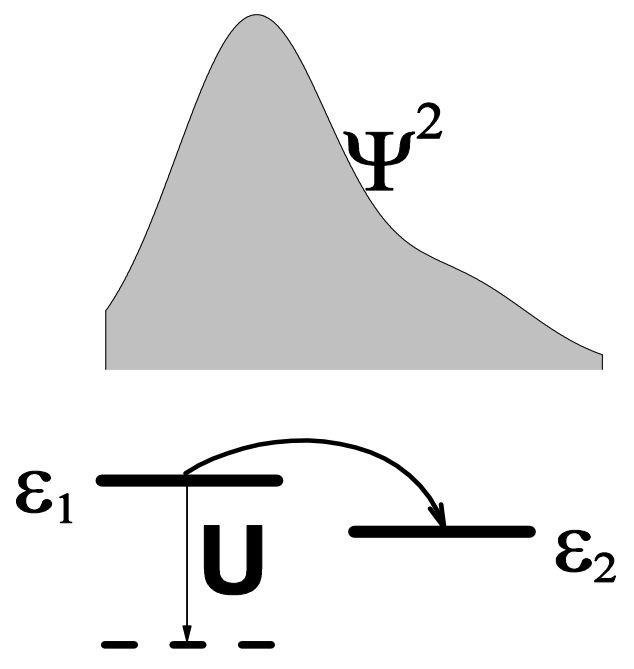

FIG. 2: By assuming that the electron is initially localized on the left (as shown schematically by the plot of $\psi^{2}$ on the top), tunnelling to the right is hindered even if $\epsilon_{2}<\epsilon_{1}$ because of the correlation energy $U$. The system has a memory of its ground state producing a hysteresis loop for the tunnelling rate between both sites. See the text for further details. 

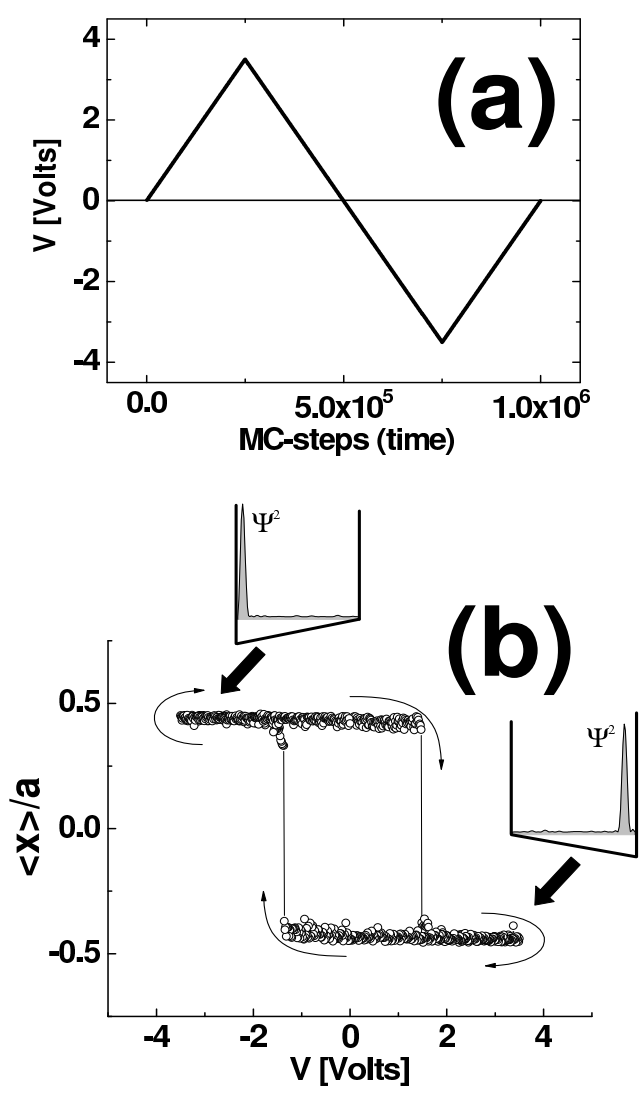

FIG. 3: Hysteresis loop for the particle in a box with a nonlinear potential (Eqn. 4). In (a) the amplitude of the potential is shown as a function of MC-steps, which play the role of time. The parameters used in the simulation were $\gamma=-1.0$, $\beta=-0.1$, and the size of the box $a=0.5$. Further details of the numerical implementation of the FMC-method can be found in Ref. 16. The particle follows adiabatically the potential changes along the MC-changes. The initial state is with the particle on the left, and it is found after a simulated annealing with 20 temperatures from $T=1$ to 0 , with $10^{4}$ thermalization cycles per temperature and 40 Fourier coefficients in total [16]. $T_{0}$ is fixed subsequently to 0.05 during the potential changes. In (b) the evolution of the expectation value of the wavepacket $\langle x\rangle / a$ is plotted as a function of the external potential. Note the presence of two very different thresholds for the tunnelling in both directions. As in Fig. 2 the reason is the competition between the intrinsic nonlinearity of the Hamiltonian (once the particle is localized on the left or right) and the applied external potential. 

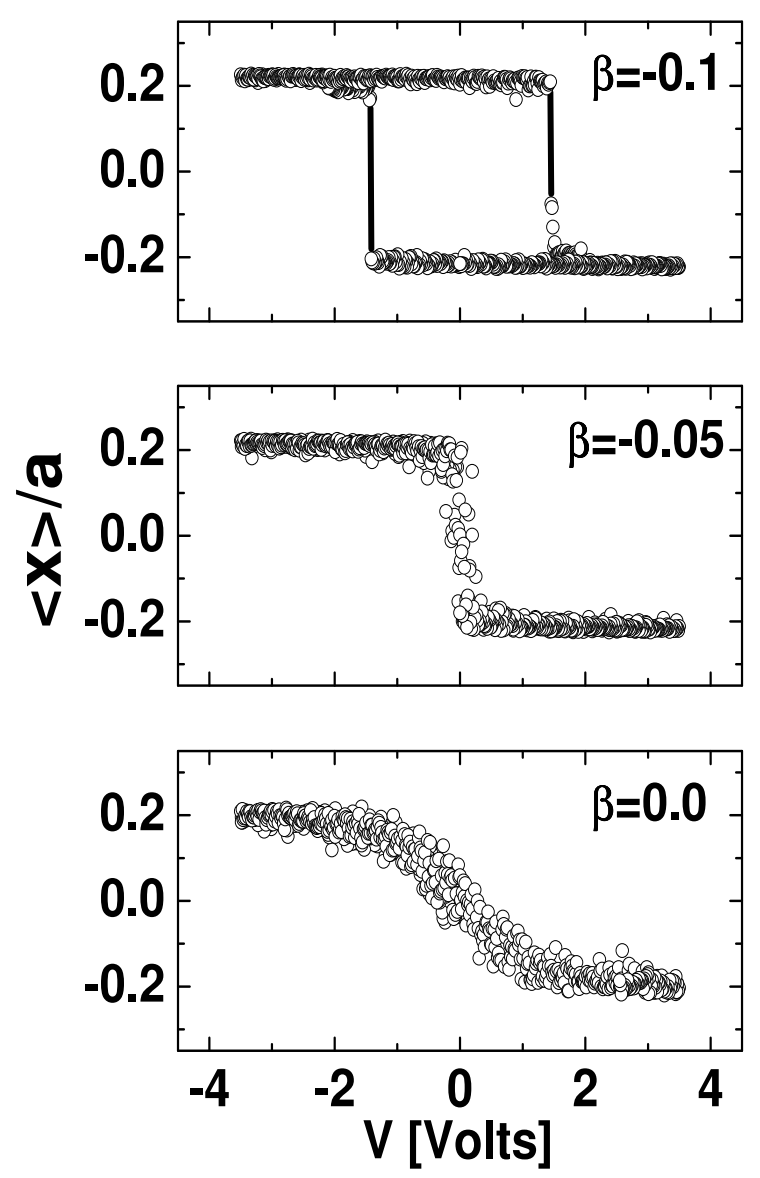

FIG. 4: Hysteresis loops for different values of the nonlinear coefficient $\beta$ in Eq. 4] For a fixed $\gamma=-1.0$, we show three examples of competition between nonlinearity and the external electric field for $\beta=-0.1$ (top), $\beta=-0.0 .5$ (middle), and $\beta=0.0$ (bottom). For $\beta=-0.05$ (middle) the coercive field on the top graph has collapsed to a very small value around $V \sim 0$. The system profits from accumulating the probability density on the left or the right of the box, but the barrier is very small and quickly overcome when the field reverses. For $\beta=0.0$ the particle follows (linearly) the electric field at low voltages, until the boundary conditions on both sides of the box start competing (and saturating) the drive of the external field. See the text for further details. 